\title{
Gains et Pertes en Traduction vus à Travers la Version Anglaise d'une si Longue Lettre de Mariama Bã
}

\author{
Oluchukwu Felicia Asadu \\ *http://dx.doi.org// 0.43 |4/ujah.vI7i3.6
}

\section{Résumé}

L'activité traduisant est un processus très compliqué qui exige la connaissance extralinguistique chez le traducteur. Ce travail est basé sur la traduction littéraire. La traduction littéraire consistedes textes littéraires que comprennent la poésie, le théâtre, et la prose. La traduction littéraire a quelques problèmes spécifiques par rapport à la traduction des textes scientifiques. Le langage littéraire est connotatifdoué de sens figuratif. La tâche d'un traducteur c'est de traduire le message en tant que tel, de la langue de départ à la langue d'arrivée afin que le lecteur du texte traduit saisisse ce que dit l'auteur. En faisant cette tâchede la langue française à la langue anglaise ou vice versa, nous constatons des phénomènes qui sont les pertes et les gains dans la traduction. Ces phénomènes sont inévitables parce que les deux langues en questions appartiennent à des communautés linguistiques différentes. Dans ce travail nous allons utiliser quelques extraits d'une si longue lettre (So Long a Letter) pour montrer d'où viennent ces phénomènes déjà mentionnés.

\section{Abstract}

Translation is a very complicated process that requires extra linguistics knowledge of a translator. This study is focused on literacy translation. Literary translation consists of literary texts such 
as poems, drama and prose. The literary translations have some specific problems when compared with the translation of scientific texts. Literary language is connotative and very rich figurative meaning. The work of a translator is to translate the message as it is from the source language to the target language so that any reader would still grab what the author said.

While translating from French to English language or vice versa, one could observe the lost and gains of meanings in the translation. These phenomena are inevitable because the two languages in question belong to different linguistic communities. In this study, we will use some extracts from So Long a Letter ( Une si longue lettre) to point out these phenomena mentioned above.

\section{Introduction}

Les phénomènes de gains et pertes ou les additions ou les omissions viennent du fait que les deux langues en questions n'appartiennent pas ni aux mêmes cultures ni aux mêmes morphologiques. C'est pourquoi Lederer $(1997: 78)$ a dit ceci : 
L'ensemble de l'extension sémantique d'un mot ne pouvant se retrouver dans la traduction, celle-ci perdrait quelque chose de l'original tandis que la précisionapportée au mot par le choix d'une de ses acceptions lui ferait au contraire gagner quelque chose.

Le cas de double traduction dans la traduction des œuvres africaines est une chose particulière. Les pays africains sont d'abord multilingues. Ils ont des langues européennes comme langues officielles. Dans les œuvres produites par les Africains comme le dirait Senghor. « Nous nous sentons en nègres mais nous exprimons en français ». Les Africains conçoivent leurs œuvres en langues africaines et les traduisent en langues européennes et le traducteur les traduit encore en une troisième langue. Beaucoup de gains et de pertes d'informations sont effectués au cours de ce processus. Parfois ni artiste ni le traducteur ne s'en rende compte.

\section{Que signifie la traduction?}

Nous ne pouvons pas traiter, de façon exhaustive, les différentes définitions de la traduction parce que beaucoup de théoriciens ont défini la traduction. Si nous considérons quelques définitions du terme traduction par quelques théoriciens nous verrons qu' Aury (1963:7) a mis son emphase sur la lisibilité d'un texte de la langue de départ à la langue d'arrivée. Elle postule que "la traduction est l'opération par laquelle un texte écrit dans une langue se trouve susceptible d'être lu dans une autre langue" Pour Nida (1963:278),

La traduction consiste à produire dans la langue d'arrivée l'équivalent naturel le plus proche du message de la langue de départ, 
d'abord quant à la signification puis quant au style.

Nida nous explique, comme nous constatons, que le traducteur doit essayer de raconter ou de rédiger dans une langue d'arrivée ce qui est rédigé dans une langue du départ. Le traducteur doit produire l'équivalent le plus proche du vouloir- dire d'un auteur chaque fois qu'il traduit.D'après Asadu (2012:14) « la traduction est l'acte de faire passer le contenu d'un message d'une langue de départ à la langue d'arrivée en gardant toutes les manifestations culturelles de message $»$.

Quant à Catford (1965:20) la traduction c'est "The replacement of textual materials in one language (source language) by equivalent textual materials in another language (target language)

Le traducteur doit garder le style de l'auteur, et le contenu du message.ll ne peut ni enrichir ni ôter la pensée de l'auteur du texte original. D'après Taber et Nida (1974:II) "la traduction est l'équivalent naturel le plus proche du message de la langue de départ à la fois en sens et en style ». C'est pertinent au traducteur de traduire le message, en tant que tel, de la langue de départ à la langue d'arrivée afin que le lecteur du texte traduit saisisse ce que dit l'auteur. Dans cette optique Paul (1992:20) a dit que :

l'objectif de la traduction est de transmettre un message. Une bonne traduction est celle qui transpose dans la langue d'arrivée l'information contenue dans la langue de départ et de l'objectif visé par l'auteur; cela implique également une définition de l'auditoire de la langue d'arrivée. 
Selon Vinay et Darbelnet (1977:24)

La traduction est l'opération qui consiste à faire passer d'une langue à une autre, tous les éléments de sens d'un passage et rien que ces éléments en s'assurant qu'ils conservent dans la langue d'arrivée, leur importance relative, ainsi que leur tonalité et en tenant compte des différences que présentent entre elles les cultures qui les correspondent respectivement, la langue de départ et la langue d'arrivée.

On voit que l'activité traduisant exige beaucoup de choses chez le traducteur. II faut que le texte traduit ressortisse le même sentiment naturel que le texte original en gardant le ton, la culture, et l'entourage des faits d'expression.

Maintes linguistes (Weinreich, 1953 ; Mounin, 1963 ; Newmark, 1982) ont proposé des définitions qui décrivent l'activité traduisant. Selon Newmark (1998:7)

Translation is a craft consisting in the attempt to replace written message and/or statement in one language by the same message andlor statement in another language

Il a fait la distinction entre la traduction (Texte écrit) et l'interprétation (oral). Pour nous, la traduction est l'acte de faire passer le contenu d'un message d'une langue de départ à la langue d'arrivée en gardant toutes les manifestations culturelles de message. On ne traduit pas pour divertir, la traduction ne se fait pas par hasard. La traduction n'est pas simplement le fait de remplacer les mots de la langue de départ par des mots équivalents dans la langue d'arrivée à l'aide d'un dictionnaire. Pour qu'on puisse traduire, on doit tenir compte du sens du texte de la langue 
de départ à la langue d'arrivée. Aussi la tonalité du message aussi bien que les éléments culturels dans les deux langues sont pertinents. Peter Newmark (198I : 165) soutient que :

La traduction est un exercice extraordinairement difficile qui exige une curiosité sans borne et pour les choses autant que pour les mots(...) des connaissances linguistiques et non linguistiques sans cesse renouvelées, une part de flair et d'imagination ainsi que de l'intelligence et par-dessus tout, du bons sens.

Ceci nous montre que la traduction est un exercice difficile. II montre aussi l'importance des connaissances linguistiques et non linguistiques pour la part d'un traducteur. Pour supporter ceci Pergnier Maurice (1978:466) a dit :

Puisque traduire ne consiste pas à commuer des systèmes linguistiques l'un en l'autre mais a transcoder les divergences de systèmes pour communiquer un (dire) singulier qui n'apporte pas à la langue qui le formalise mais qui n'emprunte les obstacles à la possibilité de traduire ne sont pas à rechercher dans la convergence ou la divergence des langues mais dans la possibilité ou l'impossibilité de trouver des formulations équivalences aux sens de message particuliers

Pour manipuler les mots, les idées dans deux langues résultent des phénomènes des gains et des pertes en traduction. 


\section{La traduction littéraire}

Le français et l'anglais peuvent se ressembler mais ne sont pas les mêmes .La situation d'Une silongue lettre se complique de plus, carla langue du texte elle-même entraine deux cultures ; la culture sénégalaise en langue française. C'est pourquoi Newmark (1989:68) a dit :

Le traducteur doit faire son possible pour opérer au même niveau que l'auteur afin de permettre à son public et à lui-même de recevoir le texte traduit au niveau de l'auteur au sein de sa propre culture.

Puisque le texte dont nous parlons est une œuvre littéraire, nous allons parler sur la traduction littéraire. La traduction littéraire est la traduction des textes opposés aux autres textes dits scientifiques ou pragmatiques En faisant la distinction entre la traduction scientifique et la traduction littéraire Wuillmart (1990:236)

Les textes scientifiques et techniques appartiennent au discours d'intention objective, analytique ou descriptive et ne laissent par conséquent à la personnalité de l'auteur que très peu d'espace... Le texte littéraire est au contraire un texte d'auteur, il baigne en plein dans la subjectivité et le résultat de l'approche d'une part artistique, de l'autre psychophysiologique d'un monde qui nous apparait précisémentà travers les lunettes d'un individu. Ce monde particulier du texte littéraire a une couleur, une ambiance, un ton, un style. 
Cette subjectivité, cette ambiance, cette couleur, ce style et ce ton sont parfois difficiles à cerner et à traduire. Pour supporter ceci Muntaner(1993:84) remarque que:

La traduction littéraire... est une œuvre pleine de servitude qui ne peut ni ne doit remplacer l'œuvre originale, mais qui dans certains cas peut atteindre un pouvoir de suggestion semblable à celui qu' existait dans la langue de départ.

Nous allons voir des critères particuliers de la traduction de la prose. Notre intérêt ici se porte sur la prose car notre œuvre est écrite en prose.McGuire (1980:116) a énuméré les six règles proposées par Belloc pour diriger le traducteur de prose, premièrement, le traducteur ne doit pas traduire mot à mot. II doit traduire le sens global du texte source. II doit comprendre le sens ou le message du texte original avant de le traduire. Deuxièmement, le traducteur va chercher l'équivalent des proverbes ou les expressions idiomatiques de la langue source et les rendre à la langue d'arrivée. Troisièmement, le traducteur doit rendre vivement le message dans la langue d'arrivée.

Quatrièmement, le traducteur doit éluder les faux - amis. C'est -à - dire les mots de langues différentes qui se correspondent par la morphologie, mais qui, ayant évolué dans deux systèmes linguistiques séparés, ont acquis des sens différents. Ils sont vraiment les faux -amis du traducteur, car ils trompent le plus souvent, des traducteurs inattentifs. Exemple :avoir de lachance :to be lucky et non pas to have chance. Cinquièmement, le traducteur doit traduire le vouloir- dire de l'auteur au lieu de traduire mot à mot ou phrase par phrase. 
Sixièmement, le traducteur ne doit jamais embellir.

Les six propositions de Belloc aident le traducteur à traduire le sens d'un texte d'une langue à l'autre. Belloc est contre la traduction mot à mot ou littérale. II partage la même idée que Danica Seleskovitch et Mariane Lederer où le sens est au premier rang. Le traducteur est libre de traduire un texte en suscitant l'idée originale du texte. Belloc est d'accord avec des modifications nécessaires pourvu qu'elles aident à atteindre l'équivalence voulue dans la langue d’arrivée.

\section{Bref aperçu biographique de l'auteur et le sommaire de l'œuvre choisie MARIAMA Bâ}

Mariama Bâ est née en 1929 à Darkar et mort en 1981 aussi au Sénégal.Elle a été élevée par ses grand parents dans un milieu musulman traditionnel et son père. Elle a été élevée par ses grands - parents dans un milieu musulman traditionnel et son père était ministre de la santé au Sénégal en 1956. Issue d'une famille traditionnelle et musulmane, Mariama Bâ intègre une école française après la mort de sa mère et se fait remarquer par des résultats distingues.

Par conséquent, elle se décide à intégrer dansl'Ecole Normale de Rufisque en 1943, qu'elle quitte munie d'un diplôme d'enseignement en 1947. Après douze ans durant lesquelles elle exerce sa profession, elle demande sa mutation au sein de l'inspection régionale d'enseignement, sa santé étant devenue fragile.

Ayant donné naissance à neuf enfants, elle obtient le divorce de son conjoint, le député Obèye Diop. Suite à son expérience du mariage, Mariama Bâ s'engage pour nombre d'associations féminines en propageant l'éducation et les droits des femmes. A cette fin, elle prononce des discours et 
publie des articles dans la presse locale.Dès sa publication en 1980, son premier roman en forme épistolaire connaît un réel succès et est retenu pour la remise du prix Noma lors de la foire du livre de francfort. Elle meurt peu de temps plus tard d'un cancer et avant la sortie de son deuxième roman. Un lycée de Dakar (La Maison d' Education Mariama Bâ) porte à ces jours son nom.Ses livres reflètent les conditions sociales de son entourage immédiat et de l'Afrique en général, ainsi que les problèmes, qui en résultent, tels que polygamie, castes, exploitation des femmes - pour le premier -, position de la famille, manque de capacité de s'adapter au nouveau milieu culturel face à des mariages interraciaux pour la deuxième roman dans la traduction. Les œuvres publiées par elle sont :

- Une si longue Lettre Roman 1979

- La Fonction Politic Des Littératures Africaines Ecrites 1981

- Le Chant Ecarlate Roman 198I.

\section{Gains et pertes en traduction}

Les gains et les pertes sont des phénomènes inévitables dans la traduction.

Au cours de la traduction le traducteur doit être explicite là où le texte source et implicite.

Parfois il y a des mots inutiles que l'auteur a utilisés comme son style qui n'ajoute aucun sens que le traducteur doit négliger en faisant sa tâche. De plus le traducteur doit ajouter ou enlever des mots, pour bien ré-exprimer le message. Cette technique selon Nida s'appelle 'Techniques of adjustment » Nida en conseillant parlant aux traducteurs a dit : 
In front of fact, what he really does, or should do is to select in each sentence the closest natural equivalent. But if the corresponding forms in the source and receptor languages are compared after such equivalents have been selected, it will be found that they conveniently fall in to such classes of modification as can be described by these terms: additions, subtractions and alterations.

Analyse de certains gains dans la traduction d'une si longue lettre

Nous allons voir quelques exemples de gains tirés dans la traduction d'œuvre choisie.

I TLD7... bouche à bouche

TLA2 ... mouth - to-mouth ressuscitation

Ici, il y a un gagne avec le mot « ressuscitation » qui rend la traduction plus explicite et montre ce que c'est vraiment donner un bouche à bouche.

\section{TLD5 Aissatou}

\section{TLA5 Dear Aissatou}

Le mot « dear » signifie que la personne est proche et aussi avec émotion mais le texte de départ est dépourvu de cette amitié et émotion.

3 TLD6 un taxi hèle ! vite !

TLA2 A taxi quickly hailed! Fast! Fast! Faster still!

Il ya la gagne dans la langue d'arrivée pour montrer l'urgence de la situation.

4 TLD9 ... je suis les allées et venues

TLA3 ... I follow the coming, and goings of people 
Ici la langue d'arrivée est plus exacte car ça montre les choses qu'elle suit parce que ça pourrait être les allées et venues des bicyclettes ou voitures.

5 TLD37 quoi, un toucouleur qui convole avec une bijoutière ( $\mathrm{pg}$ 37)

TLAI7 What, a toucouleur marrying a goldsmith's daughter?

Ici, on trouve la gagne du mot « daughter » qui rend la comprehension du texte d'arrivée plus comprehensible aux lecteurs.

6 TLD6I ... En aident à naitre des serviteurs de Mohammed TLA30 ... By helping at the birth of new followers of Mohammed, the prophet

Il y a l'addition de mot « the prophet » pour aider le lecteur à savoir de quel Mohammed il s'agit.

7.TLD 60 La petite Nabou bien prise dans sa main droite, elle reprit le chemin inverse

TLA29 Holding youngNabou's hand firmly in her right hand, she took the road back to town Ici, le chemin qu'elle reprend est clairement expliqué contrairement à ce qui a été dit dans la langue de depart. Analyse de certaines pertes dans la traduction d'une si langue lettre

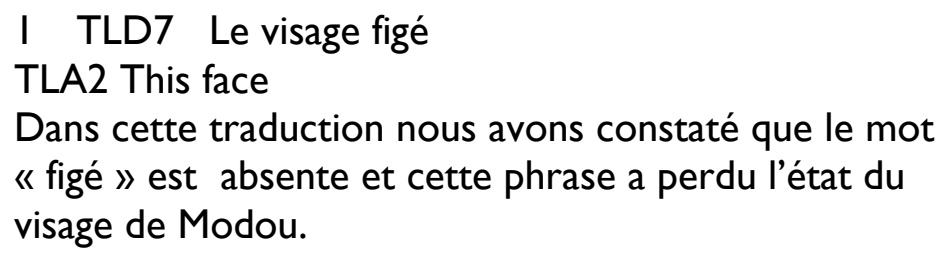
« figé » est absente et cette phrase a perdu l'état du visage de Modou. 
2 TLD29 Tu te souviens de ce train matinal qui nous emmena pour la premièretrois à Ponty - ville TLA3I For the first time to Ponty- ville ...

Ici, le traducteur a omis «train matinal» qui indiquait précisément leur moyen de transport et aussi «tute souviens » qui montrait que celle qui écrit voulait que sa lecture se souvient. Donc, la version anglaise manque de précision.

3 TLD 19 les murs qui délimitent mon horizon pendent quatre mois et dix jours. Les dix jours ne me gênent guère.

TLA8 The walls that limit my horizon, for four months and ten days do not bother me.

Dans la langue de départ le mot «dix jours» a été répété deux fois mais dans la langue d'arrivée il a été dit une fois donc il ya une manque d'emphase dans la version anglaise.

4 TLD 20 Puisse leur invocation ne rien souiller de l'état de pureté absolue ni je dois évolues.

5 TLA 9 May their evocation not soil the state of purity in which i must live.

Le mot «absolue »est absent dans la langue d'arrivée. L'auteur a précisé le degré de pureté qu'elle voulait vivre moralement mais le traducteur a évité cette précision.

6 TLD 38 Cette vie animait la flamme, tantôt rage, tantôt bleue, qui s'élevait ou se courbait, faiblissaitou s'intensifiait selon la volonté et le besoin de l'œurre.

TLA 18 This life would rise or curve, wax or wane at his command depending on what the work demanded. 
Nous avons constaté des omissions des mots suivants: « tantôtrage, tantôt bleue » qui signifient les couleurs/manifestation des flammes chez un bijoutière, et chaque couleur a quelque chose affaire pendant la production d'un bijoux en or. Donc la version anglaise reste implicite làoù la version française est implicite. Ces omissions sont les choses qui sont unique pour le métier d'un bijoutière car les différents manifestations des flammes sont très fascinant, émouvant et intéressant

7 TLD 39 - l'université aussi a ses rejets exorbitants et désespères.

TLA 18 The university has its own large number of despairing rejects.

Ici il ya l'absence du mot «exorbitants» ceci diminue l'intention de l'auteur.

Oluchukwu Felicia Asadu Department of Modern European Languages Nnamdi Azikiwe University, Awka, Nigeria 


\section{Bibliographie}

Asadu F.O. "Une traduction commentée (anglais-francais) du film « Winning Your Love par Ossy Affason Mémoire pour l'obtention du doctorat.Diss: Abia State University, Uturu, 2012.

Aury D. preface in Georges Mounin. Les problèmes théoriques de la traduction, Paris : Editions Gallimard, 1963.

Bassnett-McGuire Susan, Translation Studies. London: Methuen, 1980.

Catford, J.C. A Linguistic Theory of Translation. Oxford: Oxford University Press, 1965.

Lederer, Marianne. La traduction d'aujourd'hui,Paris : HachetteLivre, 1997.

Muntaner, J.P. "La traduction comme création littéraire" Meta 38 (4): $84-90,1993$.

Newmark, P. Approaches to Translation. Oxford: Pergamon, 1981.

- - A Textbook of Translation. New York and London: Prentice Hall, 1989.

Nida, E.A. cité par Georges Mounin Les problèmes théoriques de la traduction Paris : Gallimard, 1963.

Nida,E. and Taber, C. The Theory and Practice of Translation. Leiden: E.J. Brill, 1974.

Paul de Maeseneer: " À vous l'interne, précis de journalisme Radio", Manille 1992 : 204

Pergnier, M. Les fondements sociolinguistiques de la traduction. Paris : Honoré Champion, 1978.

Vinay, J.P and J. Darbelnet Stylistique comparée du français et de I' anglais. Paris : Didier, 1977.

Wuilmat, Françoise. " Le traducteur littéraire : un marieur empathique de cultures", Meta, xxxv, I, 1990, 236-239. 